\title{
Density Anomaly in Core-Softened Lattice Gas
}

\author{
Aline Lopes Balladares \\ Instituto de Física, Universidade Federal do Rio Grande do Sul \\ Caixa Postal 15051, 91501-970, Porto Alegre,RS, Brazil \\ Marcia C. Barbosa $\S$ \\ Instituto de Física, Universidade Federal do Rio Grande do Sul \\ Caixa Postal 15051, 91501-970, Porto Alegre,RS, Brazil
}

\begin{abstract}
A two dimensional lattice gas model with "core-softened" potential is investigated. Two liquid phases and density anomaly are found. The demixing phase transition between the two liquid phases end at a tricritical point that is also the terminus of a critical line. The density anomaly is shown to be related to this continuous line.
\end{abstract}

$\S$ To whom correspondence should be addressed (barbosa@if.ufrgs.br) 


\section{Introduction}

The phase behavior of single component systems as particles interacting via the so-called core-softened $(C S)$ potentials is receiving a lot of attention recently. These potentials exhibit a repulsive core with a softening region with a shoulder or a ramp [1]-4]. These models originates from the disere of constructing a simple two-body isotropic potential capable of describing the complicated features of systems interacting via anisotropic potentials. This procedure generates models that are analytically and computationally tractable and that one hopes are capable to retain the qualitative features of the real complex systems.

The physical motivation behind these studies is the recently acknowledged possibility that some single component systems display coexistence between two different liquid phases [3] [5]-[7]. This opened the discussion about the relation between presence of two liquid phases, the existence of thermodynamic anomalies in liquids and the form of the potential. The case of water is probably the most intensively studied. For instance, liquid water has a maximum as a function of temperature in both density and compressibility [8]. It has been proposed some time ago that these anomalies might be associated with a critical point at the terminus of a liquid-liquid line, in the unstable supercooled liquid region [5], at high pressures. This hypothesis has been supported by a varied experimental data 9] [10] that show that thermodynamic singularities are present in supercooled water, around $228 \mathrm{~K}$ and at atmospheric pressure. In spite of the limit of $235 K$ below which water cannot be found in the liquid phase without crystallization, two amorphous phases were observed at much lower temperatures [11. There is evidence, although yet under test, that these two amorphous phases are related to two liquid phases in fluid water [12] 13.

Water is not an isolated case. There are also other examples of tetrahedrally bonded molecular liquids such as phosphorus [6] 14] and amorphous silica [15] that also are good candidates for having two liquid phases. Moreover, other materials such as liquid metals [16] and graphite [17] also exhibit thermodynamic anomalies.

Acknowledging that CS potentials may engender a demixing transition between two liquids of different densities, a number of CS potentials were proposed to model the anisotropic systems described above. The first suggestion was made many years ago by Stell and coworkers in order to explain the isostructural solid-solid transition ending in a critical point [18-[19]. Debenedetti et al. [20] using general thermodynamic arguments, confirmed that a CS can lead to a coefficient of thermal expansion negative and consequently to density anomaly. This together with the increase of the thermal compressibility has been used as indications of the presence of two liquid phases [21] 22.

which may be hidden beyond the homogeneous nucleation. The difficulty with these approaches is that continuous potentials usually lead to crystallization at the region where the critical point would be expected.

In order to circumvent this problem, we study the effect of CS potentials in a lattice. Even thought the lattice is not realistic, it allows us to explore the phase space 
in a easier way. In this work we analyze a two dimensions lattice gas with nearestneighbors repulsive interactions and next-nearest-neighbors attraction. The system is in contact with a reservoir of temperature and particles. We show that this very simple system exhibits both density anomaly and two liquid phases. However, instead of having a critical point ending the coexistence line between the two liquid phases as one usually would expect, it has a tricritical point. The connection between the presence of criticality and the density anomaly is also shown.

The reminder of the paper goes as follows. In sec. 2 the model is presented and the zero temperature phases are introduced, the mean field analysis is shown on sec. 3, results from simulations are discussed in sec. 4 and our findings are summarized in sec. 5.

\section{The model and its ground state}

Our system consists of a two-dimensional square lattice with $N$ sites. Associated to each site there is an occupational variable, $\sigma_{i}$. If the site is occupied by a molecule, $\sigma_{i}=1$, otherwise $\sigma_{i}=0$. Each site interacts with its nearest neighbors with repulsive interactions and with its next-nearest neighbors with attractive interactions ( see Fig.1). Therefore the Hamiltonian of this system is given by

$$
H=-V_{1} \sum_{<i, j>} \sigma_{i} \sigma_{j}-V_{2} \sum_{<<i, j>>} \sigma_{i} \sigma_{j}
$$

where, $\langle i, j>$ represents the sum over the nearest neighbors and $\langle<i, i>>$ is the sum over the next nearest neighbors. Our system is in contact with a temperature and particle reservoirs. The grand potential is given by:

$$
\Phi=\langle\mathcal{H}\rangle-T S
$$

where $\mathcal{H}$ contains the internal energy and the contribution due to the chemical potential $\mu$, namely

$$
\mathcal{H}=H-\mu \sum_{i}^{N} \sigma_{i} .
$$

Let us now consider the ground state properties of this model. The Hamiltonian Eq. (3) allows for a number of different configurations, however, due to the lattice symmetry and the nature of interaction, just five of them might exhibit lower energy as the chemical potential is varied. They are ( see Fig.(2))

- Dense liquid (dl):

$$
\phi_{d l}=\frac{\Phi_{d l}}{N}=-2 V_{1}-2 V_{2}-\mu
$$

- Uniformly diluted liquid (udl) :

$$
\phi_{u d l}=\frac{\Phi_{u d l}}{N}=-V_{2}-\frac{1}{2} \mu .
$$




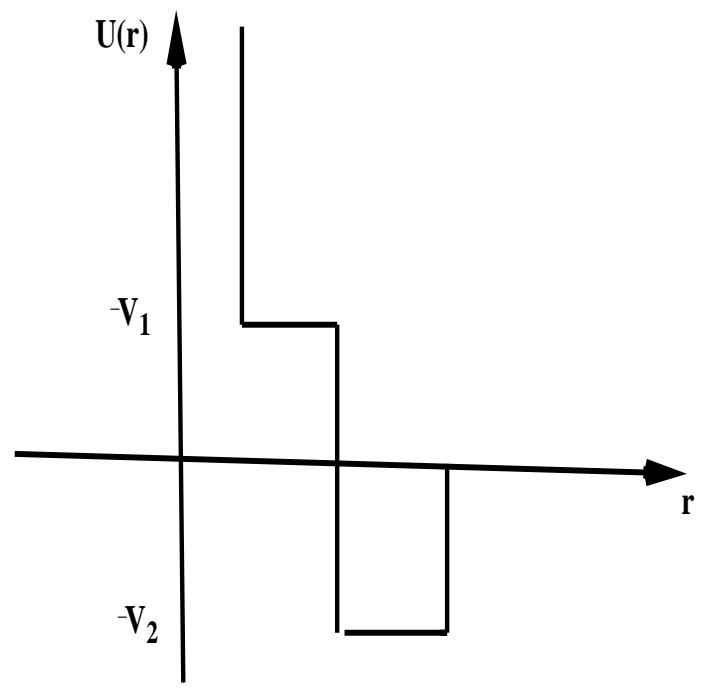

Figure 1. Schematic form of the interaction potential.

- Structured diluted liquid (sdl):

$$
\phi_{s d l}=\frac{\Phi_{s d l}}{N}=-\frac{1}{2} V_{1}-\frac{1}{2} \mu .
$$

- Semi-diluted liquid (semi-dl):

$$
\phi_{s e m i-d l}=\frac{\Phi_{s e m i-d l}}{N}=-V_{1}-V_{2}-\frac{3}{4} \mu
$$

- Gas (gas):

$$
\phi_{\text {gas }}=\frac{\Phi_{g a s}}{N}=0
$$

Here $\phi$ is the grand potential per site.

Comparing these expressions for different chemical potentials leads to the following zero temperature phase-diagram. For positive chemical potential, $\mu>>\left|V_{1}\right|$ and $\mu>>V_{2}$, the lower grand potential is associated with the dense liquid phase. As the chemical potential is reduced, the interactions between molecules become relevant. The first-neighbors repulsion together with the second-neighbors attraction favors the formation of the udl phase. At $\mu=-4 V_{1}-2 V_{2}$ there is a phase transition between the dense liquid phase and the uniformly diluted liquid phase. If the chemical potential is decreased even further, at $\mu=-2 V_{2}$ there is a transition between the uniformly diluted liquid phase and the gas phase.

\section{Mean-Field}

Now, let us exam the phase-diagram for nonzero temperatures employing a mean-field approximation. The symmetry of the different phases can be better visualized if the 


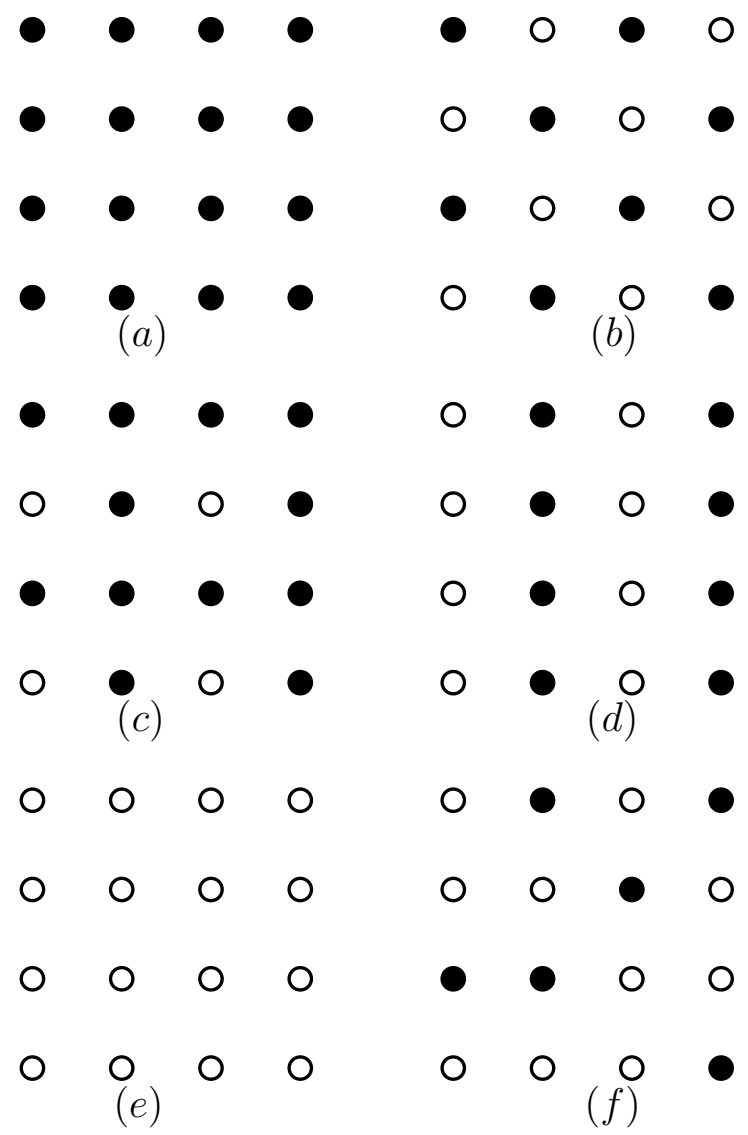

Figure 2. (a) dense liquid phase, $(b)$ uniformly dense liquid phase, $(c)$ semi-diluted liquid phase, $(d)$ structured diluted liquid phase, $e$ gas and $(f)$ fluid phase.

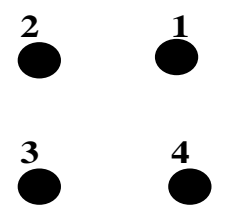

Figure 3. The sub-lattices.

square lattice is divided into four sub-lattices as illustrated in Fig.(3). In this case, the density of the sub-lattice $\alpha$ is given by:

$$
\rho_{\alpha}=\frac{4}{N} \sum_{j \in \alpha} \sigma_{j}
$$

where the sum $j \in \alpha$ is over one of the sub-lattices $\alpha=1,2,3,4$. Note that the density of each sub-lattice varies between 0 and 1 . Using the sub-lattice division, the Hamiltonian given for the Eq. (3) can be written as:

$$
\mathcal{H}=-\sum_{\alpha=1}^{4} \sum_{i \in \alpha} \mu_{\alpha}^{\text {eff }}\left(\left\{\sigma_{i}\right\}\right) \sigma_{i}
$$


where

$$
\mu_{\alpha}^{e f f}\left(\left\{\sigma_{i}\right\}\right)=\mu_{\alpha}+\sum_{\beta=1}^{4} \sum_{j \in \beta} J_{i j} \sigma_{j}
$$

is the effective chemical potential acting in an ideal sub-lattice $\alpha$. Here

$$
\mu=\frac{1}{4} \sum_{\alpha=1}^{4} \mu_{\alpha}
$$

is the chemical potential contribution due to the particles reservoir, while the second contribution in Eq. (11) is due to the interaction with other sub-lattices. The mean-field approximation we employ is to take the average of this last term, namely:

$$
\bar{\mu}_{\alpha}^{e f f}\left(\left\{\sigma_{i}\right\}\right)=\mu_{\alpha}+\sum_{\beta=1}^{4} \sum_{i \in \beta} J_{i j}\left\langle\sigma_{j}\right\rangle=\mu_{\alpha}+\sum_{\beta=1}^{4} \epsilon_{\alpha \beta} \rho_{\beta},
$$

where

$$
\rho_{\beta}=\frac{4}{N} \sum_{j \in \beta}\left\langle\sigma_{j}\right\rangle
$$

is the density of the sub-lattice $\beta$ and where

$$
\epsilon_{\alpha \beta}=\sum_{j(\neq i)} J_{i j}, \quad i \in \alpha, \quad j \in \beta
$$

is an interaction parameter. The mean-field Hamiltonian then becomes

$$
\mathcal{H}^{m f}=-\sum_{\alpha=1}^{4} \sum_{i \in \alpha}\left(\sum_{\beta=1}^{4} \epsilon_{\alpha \beta} \rho_{\beta}+\mu_{\alpha}\right) \sigma_{i}-\frac{1}{2} \sum_{\alpha=1}^{4} \frac{N}{4} \sum_{\beta=1}^{4} \epsilon_{\alpha \beta} \rho_{\alpha} \rho_{\beta}
$$

where the second term corrects for over-counting. It is straightforward to show that using Eq. (16) the mean field approximation for the grand potential per site is given by

$$
\begin{aligned}
\phi^{m f}= & -k_{B} T \ln 2-\frac{k_{B} T}{4} \ln \cosh \left[-\frac{\beta}{2} \sum_{\beta=1}^{4}\left(\epsilon_{\alpha \beta} \rho_{\beta}+\mu_{\alpha}\right)\right] \\
& -\frac{1}{8} \sum_{\alpha=1}^{4}\left(\sum_{\beta=1}^{4} \epsilon_{\alpha \beta} \rho_{\beta}+\mu_{\alpha}\right)-\frac{1}{8} \sum_{\alpha=1}^{4} \sum_{\beta=1}^{4} \epsilon_{\alpha \beta} \rho_{\alpha} \rho_{\beta} .
\end{aligned}
$$

The sub-lattice density can be derived both from Eq. (14) and from the mean field grand potential by deriving it with respect to the chemical potential, namely

$$
\rho_{\alpha}=-4\left(\frac{\partial \phi^{m f}}{\partial \mu_{\alpha}}\right)_{T, \mu_{\alpha \neq \beta}} \alpha=1, \ldots, 4 .
$$

In both cases, the derivation leads to;

$$
\rho_{\alpha}=-\frac{1}{2} \tanh \left[\frac{\beta}{2} \sum_{\beta=1}^{4}\left(\epsilon_{\alpha \beta} \rho_{\beta}+\mu_{\alpha}\right)\right]-\frac{1}{2}, \quad \alpha=1,2,3,4 .
$$

The phase-diagram, illustrated in Fig.(4), is obtained from solving Eq. (19) and Eq. (17) for different temperatures and chemical potentials. Here $V_{1}=-1$ and $V_{2}=1$ are fixed. Different choices of the interaction parameters do not change the phasediagram qualitatively. At high temperatures, all the sub-lattice densities are $\rho_{\alpha}=1 / 2$ 


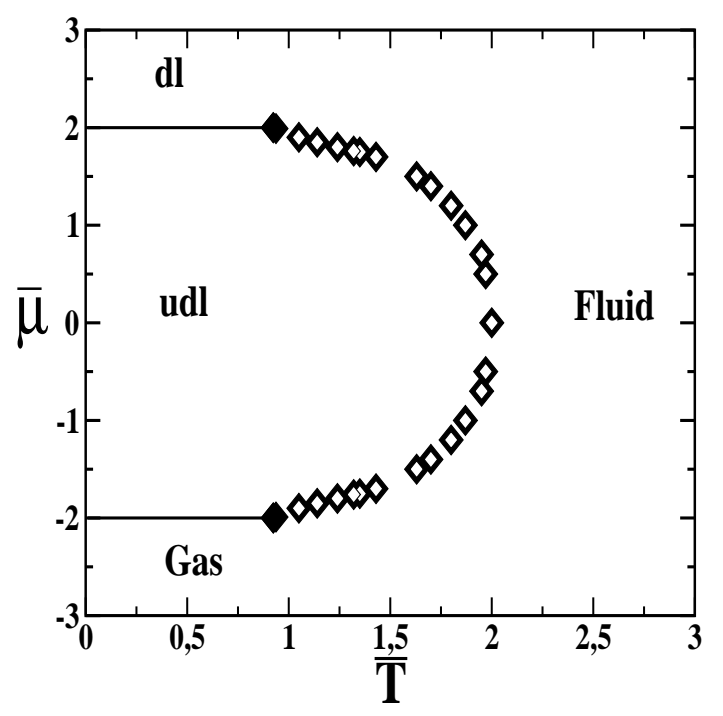

Figure 4. Phase Diagram for $V_{2} /\left|V_{1}\right|=1$. The empty diamonds are continuous lines, the solid lines are first order transitions and the filled diamonds are the tricritical points.

and the system is in the fluid phase. As the temperature is decreased at high chemical potential, $\bar{\mu}=\mu /\left|V_{1}\right|>2$, the sub-lattices become full and the system goes from the fluid to the dense liquid phase. For negative chemical potentials, $\bar{\mu}<-2$, the sub-lattices get empty, $\rho_{\alpha}=0$, and the system goes from the fluid to the gas phase. Between these two limits, $2>\bar{\mu}>-2$, as the temperature is decreased two opposite sub-lattices become empty while the other two get full. The system goes from the fluid phase to the udl phase through a continuous phase transition, $\bar{T}_{c}(\bar{\mu})$. The low temperature coexistence lines between the udl phase and the dl phase and between the udl and the gas phase are obtained by comparing the grand potentials per particle, Eq. (17), of these phases. The udl-dl phase boundary at $\bar{\mu}=2$ and the udl-gas coexistence line at $\bar{\mu}=-2$ meet the critical line $T_{c}(\bar{\mu})$ at two tricritical points at $\left(\bar{T}_{t}=0.924, \bar{\mu}_{t}=2\right)$ and $\left(\bar{T}_{t}=0.924, \bar{\mu}_{t}=-2\right)$ respectively.

\section{Monte Carlo Simulation}

The rather simple mean field approach employed in the previous session is unable to account for the density anomaly. For investigating the possibility of a density anomaly in our potential, Monte Carlo simulations in grand canonical ensemble were performed. The Metropolis algorithm was used to study square $L x L$ lattice and $V_{2} /\left|V_{1}\right|=1$. Different system sizes $L=10,20,30,50$ were investigated. Equilibration time was 1000000 Monte Carlo time steps for each lattice site.

The Monte Carlo simulations at fixed chemical potentials give the following results. 


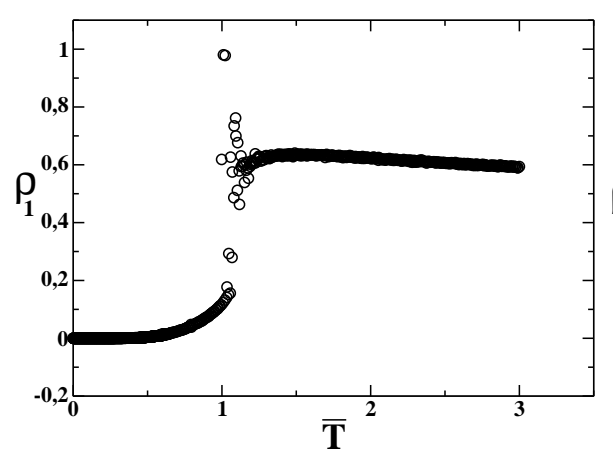

(a)

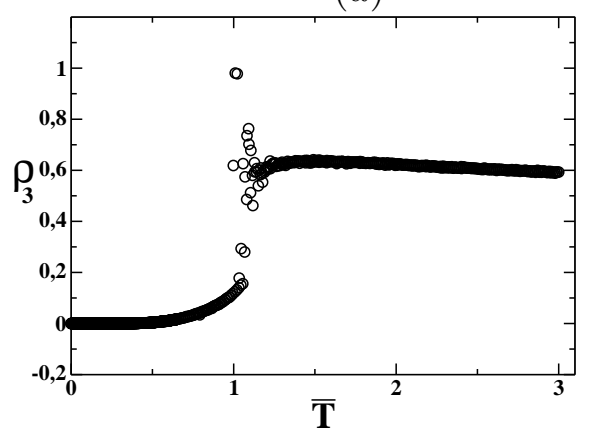

(c)

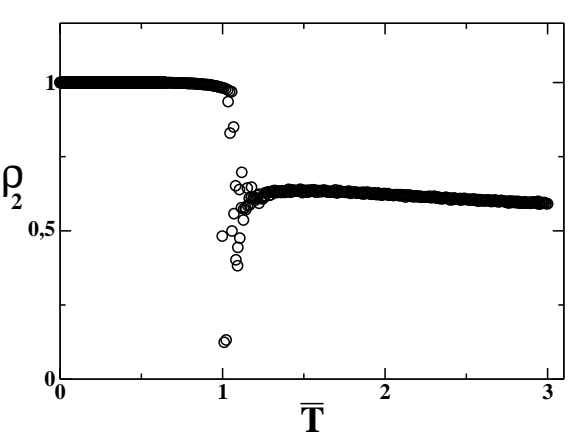

(b)

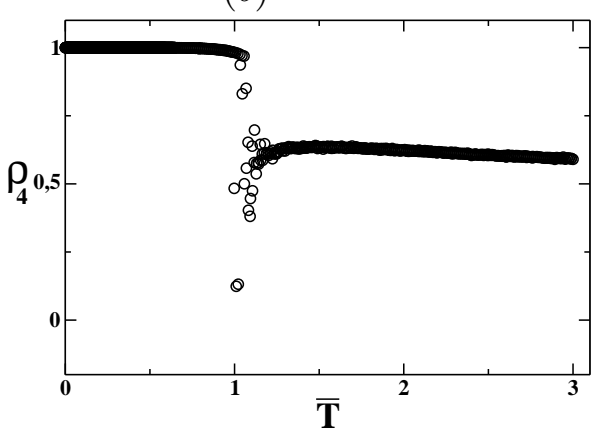

$(d)$

Figure 5. Densities of the $(a)$ sub-lattice 1, $(b)$ sub-lattice 2, $(c)$ sub-lattice 3 and $(d)$ sub-lattice 4 for the lattice $20 x 20$ at $\bar{\mu}=1.2$.

At high temperatures, the density of each sub-lattice is around $\rho_{\alpha}=1 / 2$ with sites randomly occupied in each sub-lattice, so the system is in the fluid phase. As the temperature is decreased at a fixed chemical potential, $\bar{\mu}>2$, the density of each sublattice increases continuously to one. The system goes from the fluid phase to the dense liquid phase with no phase transition. If the system is cooled from the fluid phase at low chemical potential, $\bar{\mu}<-2$, the density decreases continuously from the fluid phase to the gas phase with no phase transition. As the temperature is decreased at fixed chemical potential, $-2<\bar{\mu}<2$, one finds that two opposite sub-lattices ( 1 and 3 for example) become full while the other two sub-lattices ( 2 and 4) get empty what characterizes the udl phase. Fig.(5) shows that at $\bar{\mu}=1.2$ the density of each sub-lattice jumps from the fluid phase value to the udl phase value at the critical temperature $\bar{T}_{c}(\bar{\mu}) \equiv T_{c} /\left|V_{1}\right|=1.18$ Simulations for various fixed chemical potentials, allow us to find the critical line $\bar{T}_{c}(\bar{\mu})$.

In principle, the fluctuations observed in Fig.(5) suggest that the transition at $\bar{T}_{c}(\bar{\mu})$ is continuous. This assumption is supported by the increase in the specific heat at a fixed chemical potential. Fig.(6) illustrates this increase at $\bar{T}_{c}(\bar{\mu})=1.18$ for $\bar{\mu}=1.2$. 


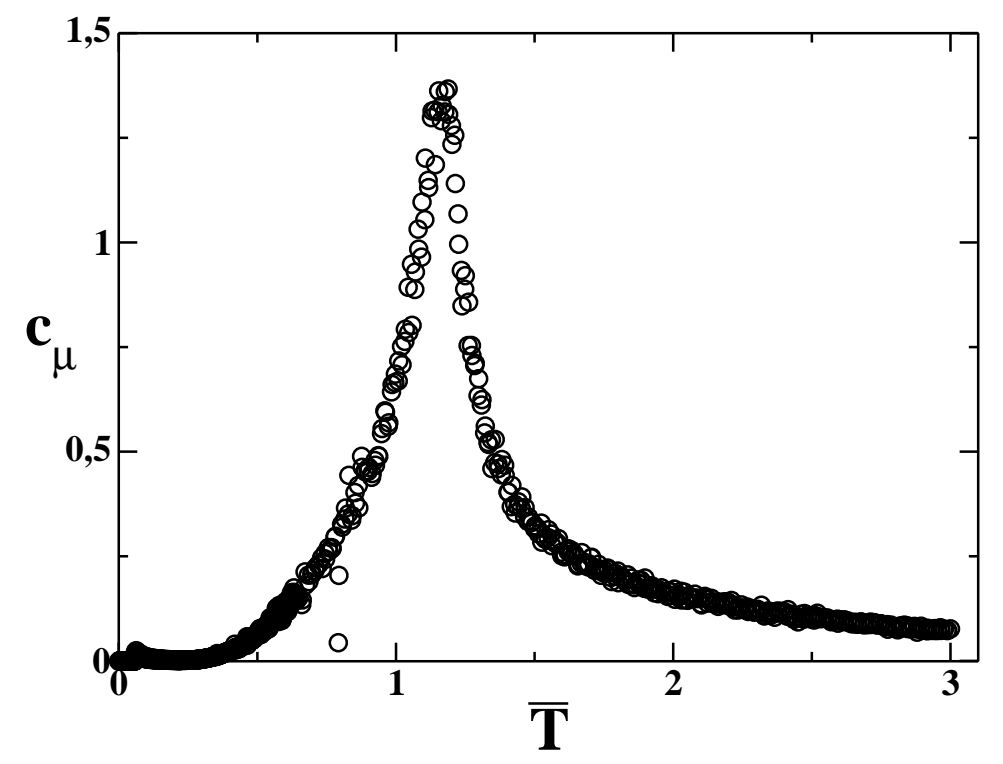

Figure 6. Specific heat for the lattice $20 x 20$ and $\bar{\mu}=1.2$.

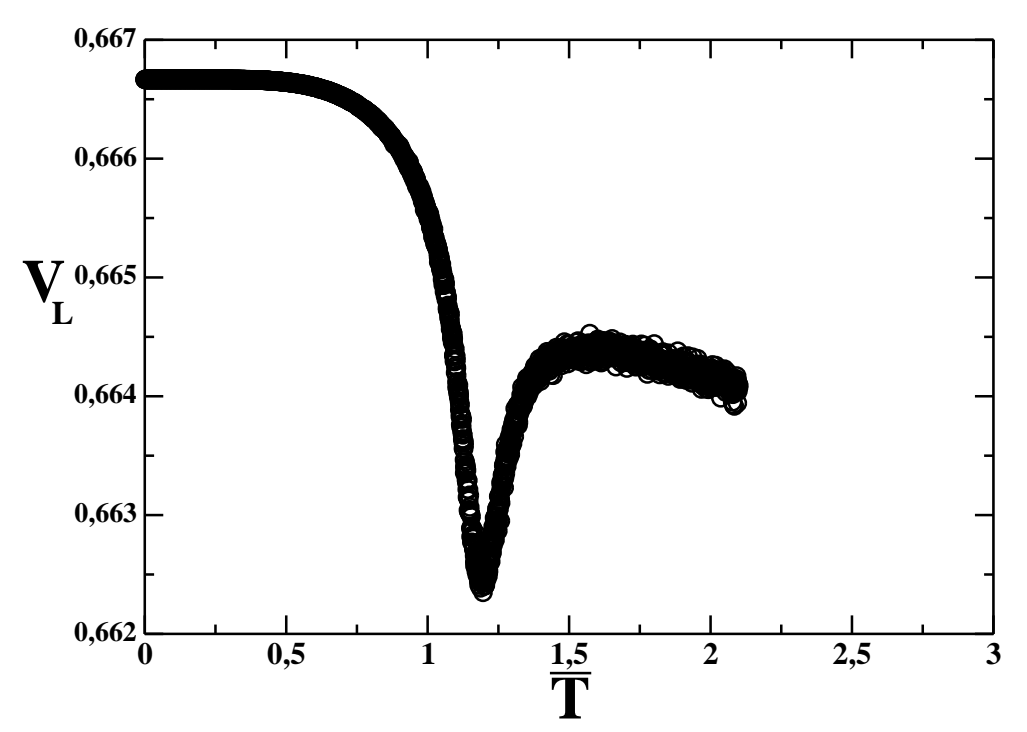

Figure 7. Fourth-order Binder's cumulant for the lattice $20 x 20$ and for $\bar{\mu}=1.2$.

The presence of a minimum in the fourth-order Binder cumulant given by [23]:

$$
V_{L}=\frac{1-\left\langle\mathcal{H}^{4}\right\rangle}{3\left\langle\mathcal{H}^{2}\right\rangle^{2}}
$$

is an indication of criticality. Fig.(7) shows $V_{L}$ for $\bar{\mu}=1.2$ obtained from our simulation. The minimum at $\bar{T}_{c}(\bar{\mu})=1.1787$ is a sign of the presence of criticality. The hypothesis that the transition at $\bar{T}_{c}(\bar{\mu})$ is first-order is eliminated by computing the energy histogram shown in Fig.(8). If the transition would be first-order, two distinct peaks should be present.

In order to check the location of the coexistence lines observed by the mean- 


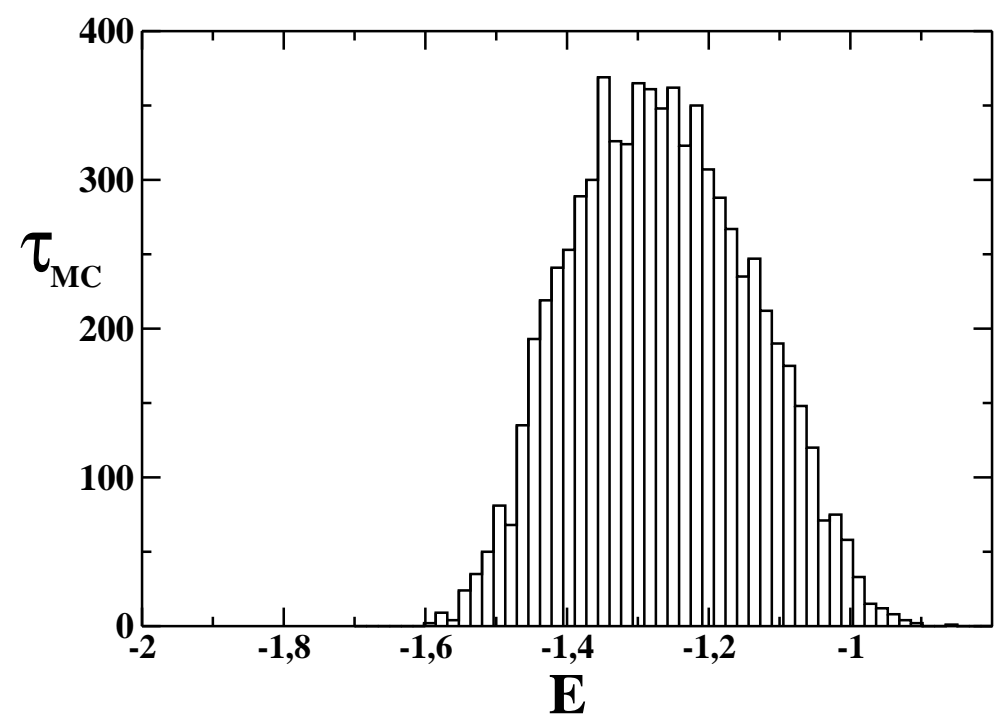

Figure 8. Energy versus Monte Carlo steps, $\tau_{M C}$ for the lattice $20 x 20, \bar{\mu}=1.2$ and $\bar{T}=1.18$. The presence of only one peak characterizes a continuous transition.

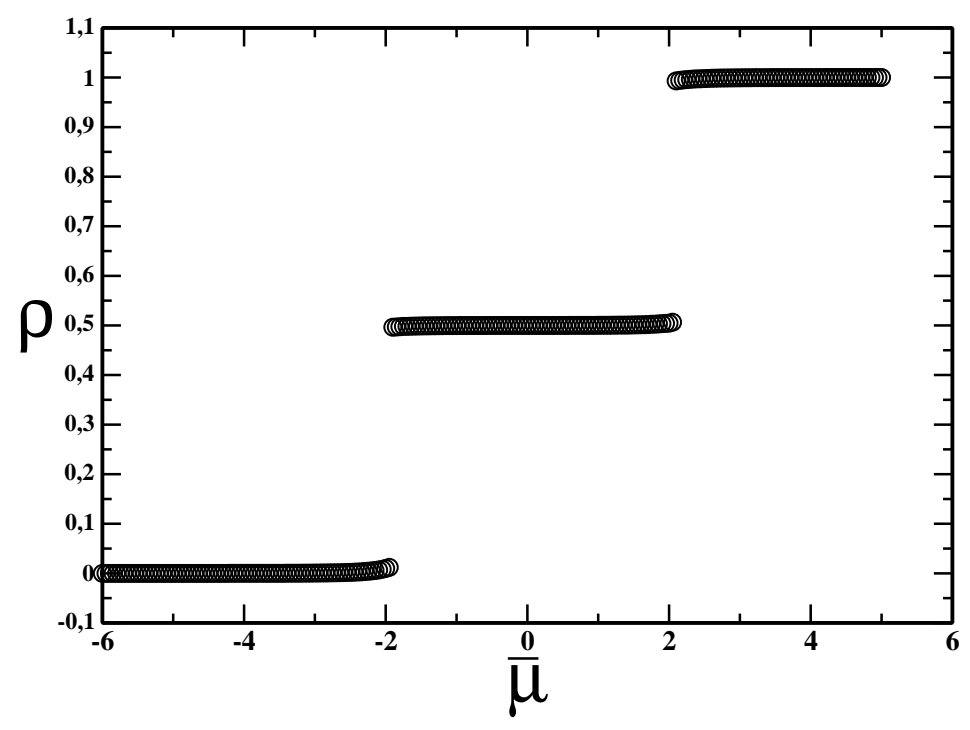

Figure 9. $\rho . v s . \bar{\mu}$ : the first-order transitions between the gas-udl and udl-dl are illustrated.

field analysis, simulations varying the chemical potential at a fixed temperature were performed. The results are shown in Fig.(9). For temperatures within the interval $0<\bar{T}<0.5$, as the chemical potential is increased the system exhibits two first-order phase transitions, one between the gas and udl phase and another between the udl and the dense liquid phase.

The total density of the system at fixed chemical potential is illustrated in Fig.(10). Differently from the mean-field result, simulations show an anomalous behavior of the density, instead of being monotonic with temperature, the density at positive chemical 


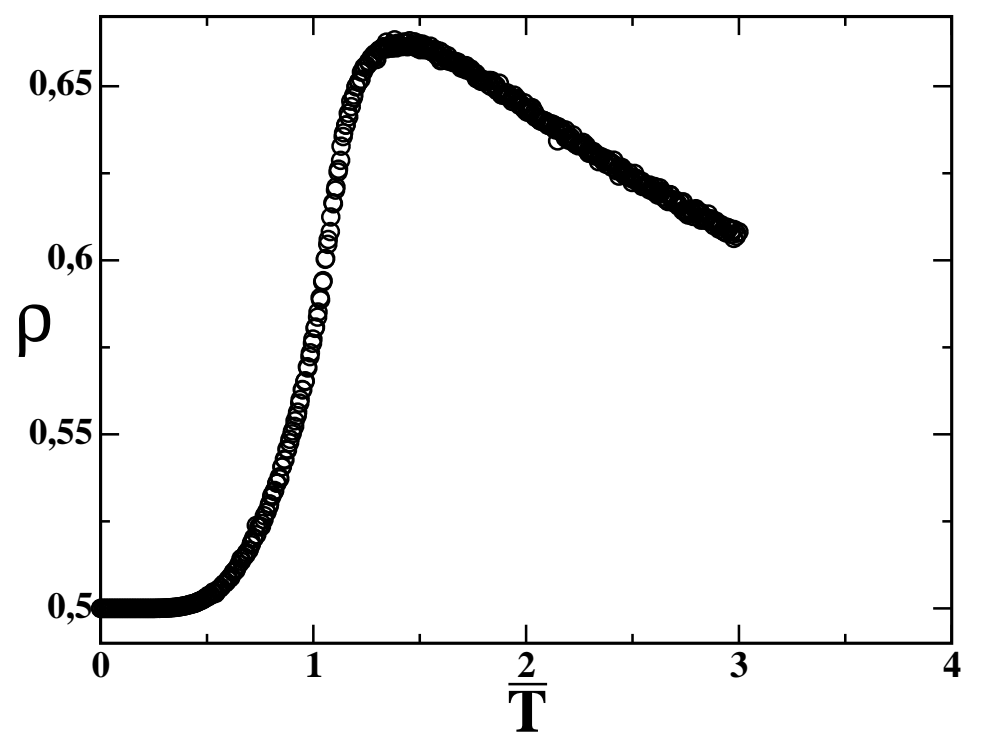

Figure 10. $\rho . v s . T$ : the maximum in the density for the lattice $20 x 20$ and $\bar{\mu}=1.4$ is shown.

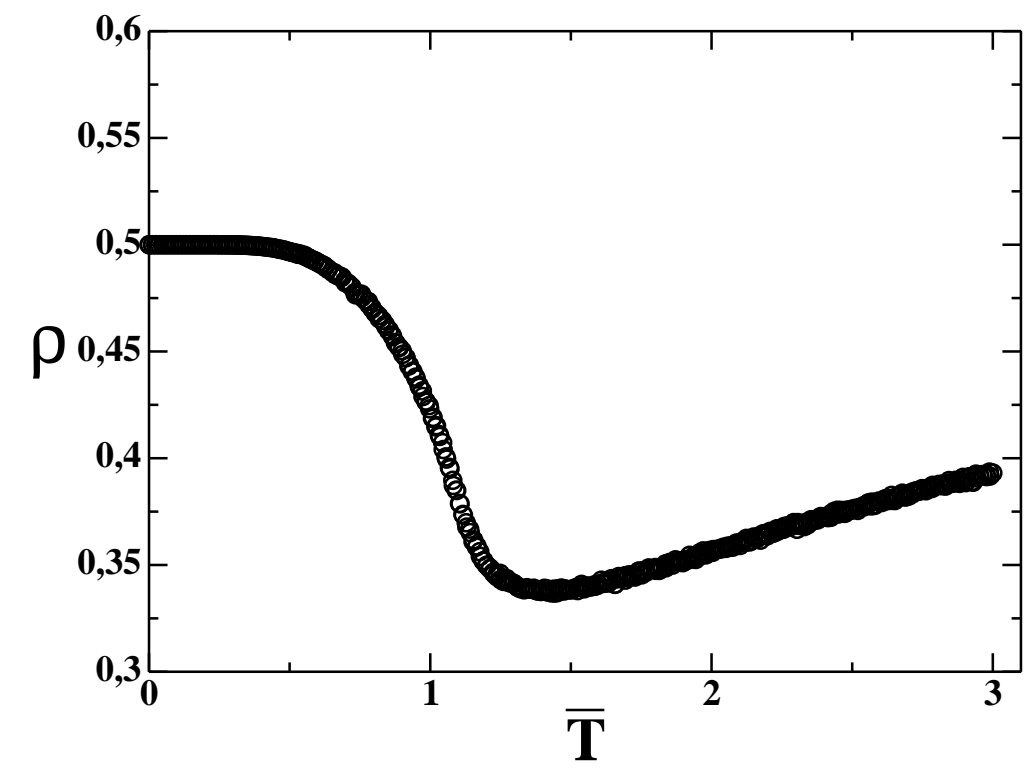

Figure 11. $\rho . v s . T$ : the minimum in the density for the lattice $20 x 20$ and chemical potential $\bar{\mu}=-1.4$ is shown.

potential increases as the temperature is decreased, it has a maximum at a temperature of maximum density $\left(\bar{T}_{T M D}(\bar{\mu})\right)$ and then decreases. For negative chemical potentials, the complementary effect is shown in Fig.(11), defining a temperature of minimum density $\left(\bar{T}_{T m D}(\bar{\mu})\right)$.

Summarizing the results discussed above, the $\mu . v s . T$ phase-diagram is shown in Fig.(12). The critical line that separates the fluid from the uniformly diluted phase joint the phase boundaries between the uniformly diluted phase and the dense liquid and gas 


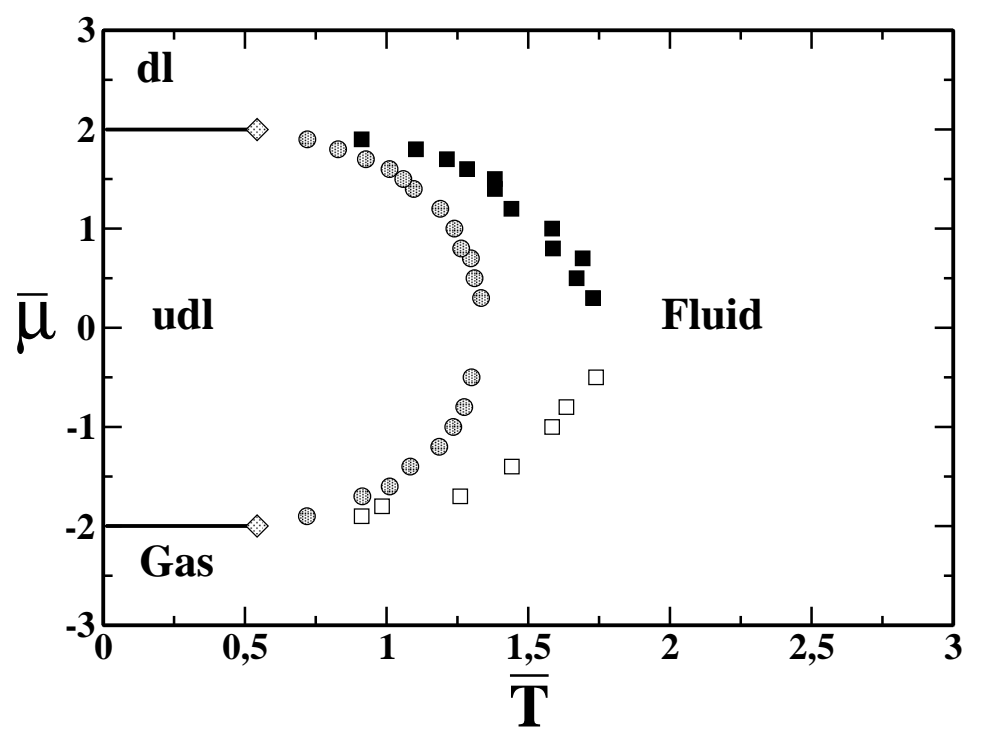

Figure 12. $\bar{\mu} \cdot v s \cdot \bar{T}$ phase-diagram for the lattice $20 x 20$. The solid lines are the firstorder transitions, the circles are the critical line, the filled squares are temperatures of maximum density $(T M D)$, the empty squares are the temperatures of minimum density $(T m D)$ and the diamonds are the tricritical points.

phases at symmetric tricritical points at $(\bar{\mu}=2, \bar{T}=0.5237)$ and $(\bar{\mu}=-2, \bar{T}=0.5237)$ respectively. The lines of temperature of maximum density and minimum density at constant chemical potential are shown.

The p.vs.T phase diagram (see Fig.(13)) is constructed by numerically integrating simulations at constant pressure. It exhibits two liquid phases, a critical line and two tricritical points like in Fig.(12). Close to the critical line, there is a line of temperature of maximum density at constant pressure.

From finite size scaling analysis [24], it is possible to estimate the critical temperature of an infinite system by the following expression:

$$
\bar{T}_{0}(\bar{\mu})=T_{c}(\bar{\mu})\left(1+x_{0} L^{-1 / 2}\right)
$$

where $\bar{T}_{0}(\bar{\mu})$ is the critical temperature of the finite system, $T_{c}(\bar{\mu})$ is the critical temperature of infinite system and $x_{0}$ a parameter.

Fig.(14) shows the critical temperatures of the finite systems as a function of the system size $L$. The value of $\bar{T}_{0}$ was obtained from two different methods: the maximum of the specific heat at a fixed chemical potential $\bar{\mu}=1.7$ and the minimum of the Binder cumulant at the same chemical potential. The extrapolated critical temperatures are $\bar{T}_{c}(\bar{\mu})=0.92079$ and $\bar{T}_{c}(\bar{\mu})=0.92543$ respectively. The difference between these two values is within our error bars of our simulations. 


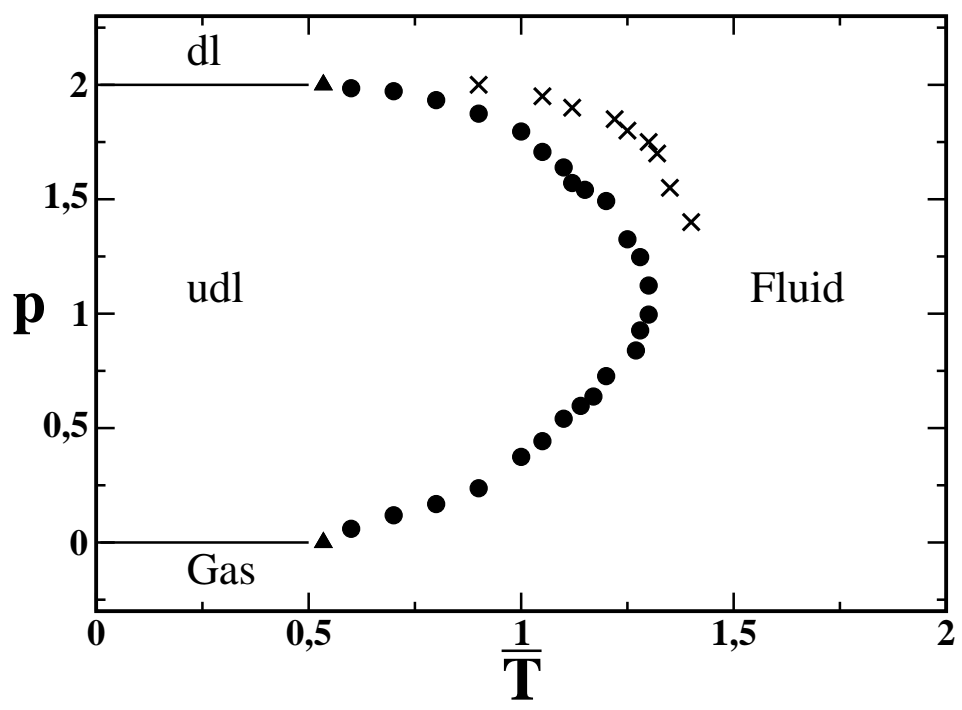

Figure 13. p.vs. $\bar{T}_{0}$ phase-diagram for the $20 x 20$ lattice. The solid lines are first-order transitions, the filled circles are the critical line, the crosses are the TMD at constant pressure line and the filled triangles are the tricritical points.

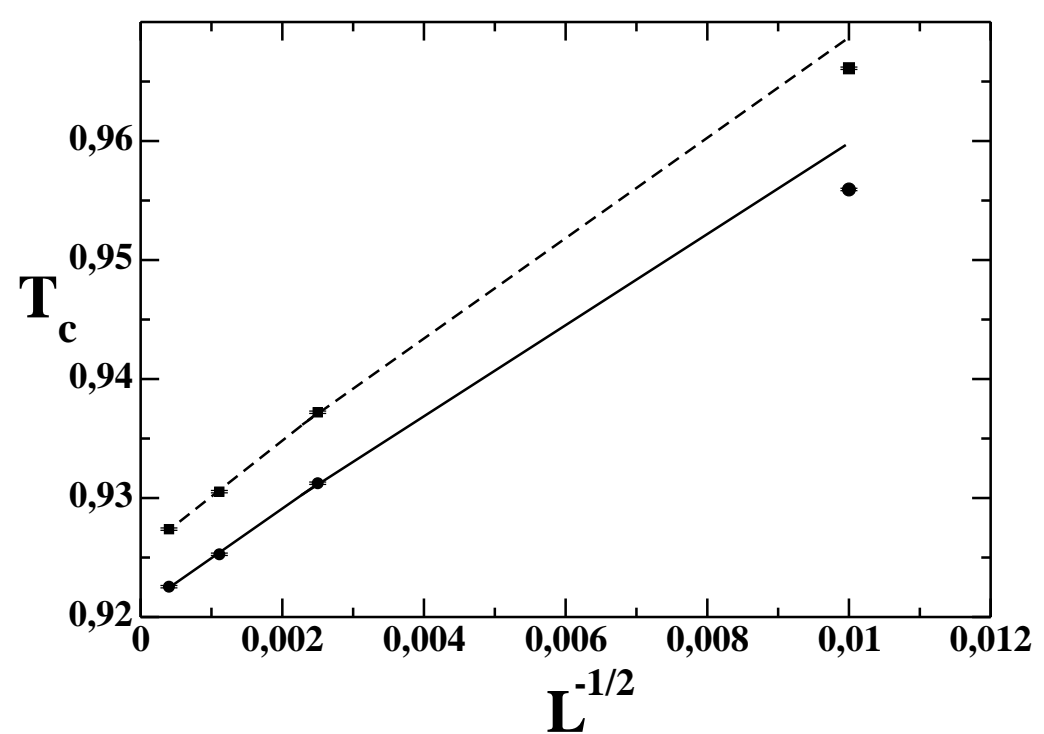

Figure 14. Minimum of the Binder cumulant (dashed line) and maximum of the specific heat (solid line) as a function of the system size $L=10,20,30,50$ for a fixed chemical potential $\bar{\mu}=1.7$.

\section{Conclusions}

In this paper the phase-diagram of a two dimensional lattice gas model with competing interactions was investigated by mean-field analysis and Monte Carlo simulations. It was shown that this system exhibits two liquid phases and a line of density anomalies.

The relation between the criticality and the density anomaly in this model goes as follows. The two liquid and the gas phases appear as a result of two competing 
interactions: the softened core that favors the formation of the uniformly dilute liquid phase and the chemical potential that induces the system to become completely filled or empty. For each soft-core interaction parameter, there is a limit chemical potential beyond which the system is in the dense liquid phase and another negative chemical potential beneath which the system is in the gas phase. The double criticality arises from the competition between $\bar{\mu}$ and $V_{1}$.

In systems dominated by short-range attractive forces the density increases on cooling. For the soft-core potential studied here similar behavior is only observed when the short-range repulsion becomes irrelevant ( high temperatures, $\bar{\mu}>2$ and $\bar{\mu}<-2$ ).

For $2>\bar{\mu}>-2$, the soft-core repulsion prevents the density to increase to one as the temperature is decreased. Therefore, the same competition responsible for the appearance of two liquid phases leads to the density anomaly. Similar analysis can be made when the pressure instead of the chemical potential is kept constant.

The presence of a critical line instead of a single critical point as one could generally expect [5] is not surprising. Due to the lattice structure, the udl is not one single phase but a region where two different phases coexist: alternating empty/full rows and alternating empty/full columns. These two phases become critical together with the dense liquid phase at the tricritical point that is also the locus the where critical line ends. The link between competing interactions and the presence of density anomaly and two liquid phases is being tested in other simple models [25] [26].

\section{Acknowledgments}

This work was supported by the Brazilian science agencies CNPq, FINEP, Capes and Fapergs.

[1] E. A. Jagla, J. Chem. Phys. 111, 8980 (1999).

[2] E. A. Jagla, Phys. Rev. E 63, 061509 (2001).

[3] G. Franzese, G. Malescio, A. Skibinsky, S. V. Buldyref and H. E. Stanley, Nature 409, 692 (2001).

[4] N. B. Wilding and J. E. Magee, Phys. Rev. E 66, 031509 (2002).

[5] P.H. Poole, F. Sciortino, U. Essmann, and H. E. Stanley, Nature 360, 324 (1992); Phys. Rev. E 48, 3799 (1993); F. Sciortino, P.H. Poole, U. Essmann, and H.E. Stanley, Ibid 55, 727 (1997); S. Harrington, R. Zhang, P.H. Poole, F. Sciortino, and H.E. Stanley, Phys. Rev. Lett. 78, 2409 (1997).

[6] Y. Katayama, T. Mizutani, W. Utsumi, O. Shimomura, M. Yamakata and K. Funakoshi, Nature 403, 170 (2000).

[7] J. N. Glosli and F. H. Ree, Phys. Rev. Lett. 82, 4659 (1999).

[8] P. G. Debenedetti, Metastable Liquids: Concepts and Principles, Princeton University Press, Princeton, 1996.

[9] O. Mishima and H. E. Stanley, Nature 396, 329 (1998).

[10] R.J. Speedy and C.A. Angell, J Chem Phys 65, 851 (1976).

[11] O. Mishima, L. D. Calvert and E. Whalley, Nature 310, 393 (1984).

[12] R. S. Smith and B D. Kay, Nature 398, 788 (1999).

[13] O. Mishima and Y. Suzuki, Nature (London) 419, 599 (2002); R. Martonak, D. Donadio and M. Parrinello, Phys. Rev. Lett. 92, 225702 (2004). 
[14] G. Monaco, S. Falconi, W.A. Crichton and M. Mezouar, Phys. Rev. Lett. 90, 255701 (2003).

[15] D.J. Lacks, Phys. Rev. Lett. 84, 4629 (2000).

[16] P. T. Cummings and G. Stell, Mol. Phys. 43, 1267 (1981).

[17] M. Togaya, Phys. Rev. Lett 79, 2474 (1997).

[18] P. C. Hemmer and G. Stell, Phys. Rev. Lett. 24, 1284 (1970); G. Stell, P. C. Hemmer, J. Chem. Phys. 56, 4274 (1972).

[19] J. S. Hoye, P. C. Hemer, Physica Norvegica 7, 1 (1973).

[20] P. G. Debenedetti, V. S. Raghavan, S. S. borick, J. Phys. Chem. 95, 4540 (1991).

[21] M. R. Sadr-Lahijany, A. Scala, S. V. Buldyrev, and H. E. Stanley, Phys. Rev. Lett. 81, 4895 (1998 ) .

[22] A. Scala, F. W. Starr, E. La Nave, H. E. Stanley and F. Sciortino, Phys. Rev. E 62, 8016 (2000).

[23] Murty S. S. Challa, D. P. Landau and K. Binder, Phys. Rev. B 34, 1841 (1986).

[24] M. E. Newman and G. T. Barkema, Monte Carlo methods in Statistical Physics, Oxoford, Clarendon Press, 1999.

[25] Vera B. Henriques and Marcia C. Barbosa, "Liquid Polymorphism and Density Anomaly in a Lattice Gas Model", submitted.

[26] Alan B. de Oliveira and Marcia C. Barbosa, "Density Anomaly in Lattice Gas Model with Two Competing Interactions", submitted. 